\title{
Analyzing Social Interactions: The Promises and Challenges of Using Cross Recurrence Quantification Analysis
}

\author{
Riccardo Fusaroli, Ivana Konvalinka and Sebastian Wallot
}

\begin{abstract}
The scientific investigation of social interactions presents substantial challenges: interacting agents engage each other at many different levels and timescales (motor and physiological coordination, joint attention, linguistic exchanges, etc.), often making their behaviors interdependent in non-linear ways. In this paper we review the current use of Cross Recurrence Quantification Analysis (CRQA) in the analysis of social interactions, and assess its potential and challenges. We argue that the method can sensitively grasp the dynamics of human interactions, and that it has started producing valuable knowledge about them. However, much work is still necessary: more systematic analyses and interpretation of the recurrence indexes and more consistent reporting of the results,more emphasis on theory-driven studies, exploring interactions involving more than 2 agents and multiple aspects of coordination, and assessing and quantifying complementary coordinative mechanisms. These challenges are discussed and operationalized in recommendations to further develop the field.
\end{abstract}

\section{Introduction}

Human beings possess an impressive ability to coordinate their actions and goalsfrom the small scale of the dyad, all the way up to the largest scales that span social groups and societies. We coordinate while dancing [1], we excel at managing complicated traffic situations [2], we effectively share information and make important collective decisions with colleagues and family members [3], and we even organize larger scale processes to sail massive battleships [4] and coordinate complex political systems [5]. Increasingly, the study of human cognition and behavior is focusing on the ways we interact to create such cognitive and behavioral synergies: the ways

R. Fusaroli $(\bowtie) \cdot$ I. Konvalinka $\cdot$ S. Wallot

Aarhus, Denmark

e-mail: fusaroli@gmail.com 
people effectively engage each other through language and actions, managing to coordinate their cognitive processes and even physiology, in order to create rapport, share information and achieve joint goals [6, 7]. Much is at stake in this enterprise, since social interactions do not only lie at the core of our private and economic wellbeing, but are also thought to be one of the most crucial aspects in mental health and healthy development [8].

In this article we discuss some of the crucial challenges in analyzing social interactions. Within this framework we introduce Cross Recurrence Quantification Analysis (CRQA). We systematically review the studies that have employed CRQA to analyze the unfolding of social interactions and the results they report. ${ }^{1}$ The aim is to critically evaluate the potential of the method in assessing the quality of various interactions. Finally we discuss the challenges still to be faced and we provide recommendations to further develop the field.

\section{Analyzing Social Interactions: The Challenges}

When compared to the classical research paradigms in cognitive psychology, the study of human interactions presents tough methodological challenges to the cognitive scientist [9]. In an interaction there are at least two agents, most often employing several expressive modalities (e.g. words, prosody, gestures, posture, etc.) and continually influencing each other, in ways that are difficult to capture when the individual behaviors are analyzed separately [10-12]. Much of the research in social cognition has either focused on the quantification of intra-personal phenomena, or confined research to ask very basic questions, such as "how strongly aligned are the interacting agents?" [13], assuming homogeneous and stationary behavior across the whole episode of interest. This approach has produced valuable insights into human interactions: by measuring how similar the frequency of given behaviors are between interacting individuals, it has been shown that people engaged in interactions tend to imitate each other's gestures [14] and align their lexicon and syntax [15, 16].

However, interactions are more complex than that. Doing the same thing is not enough to make an effective joint decision, or to coordinate on who is going to pass through a narrow train corridor first. Much in interactions is about not doing the same thing, establishing differential roles (e.g. a leader and a follower) [17-19], complementing each other (e.g. following a question with an answer, or producing complementary actions to better coordinate a task) [20,21]. This is especially emphasized through the use of culturally evolved routines (e.g. how to greet, how to apologize and how to repair misunderstandings) and scripts which enable to maximize efficiency (e.g. military rules of conduct and codes for emergencies) [10, 22].

\footnotetext{
${ }^{1}$ The review was accomplished by searching for "cross recurrence" and "crqa" on PubMed, Google Scholar and Web of Science (on October $1^{\text {st }} 2013$ ) and then manually selecting the articles analyzing social interactions. We followed up on the bibliography of these articles to individuate further relevant ones. The resulting list counts 41 articles, 34 of which reporting empirical studies and the rest being reviews or method papers. To these we added 6 submitted, but not yet published papers.
} 
In addition, interactions tend to behave in non-stationary ways. For example, imagine the escalation inherent in a heated discussion where interlocutors keep topping each other's voice or periods of high engagement followed by disengagement in attacker-defender exchanges in sports [23]. This implies that the statistical measures of moment-to-moment interactions (such as the mean and the range of values) might vary over time, defying the assumptions of linear methods [24, 25], and poses important methodological problems for the field of quantitative interaction studies.

We introduce CRQA as a way to cope with at least some of theseissues, and show how this non-linear method has proven suitable in quantifying many non-stationary coordinative patterns across various modalities and interactions, as well as discuss the challenges that it still faces.

\section{An Introduction to Cross Recurrence Quantification Analysis}

CRQA was introduced by Zbilut et al. [26] as an extension of Recurrence Quantification Analysis (RQA, for a comprehensive discussion of the method, see [27]). RQA is a more articulated non-linear equivalent of auto-correlation. It reconstructs the dynamical system underlying a time-series, maps its possible states and quantifies the trajectory of the system through these states [28]. RQA thus grants quantitative indexes of how strongly patterned the behavior of a system is, which kinds of patterns are repeated and how complex/flexible the repetitionsare. CRQA could analogously be defined as a more sophisticated non-linear equivalent of cross-correlation: it quantifies the strength, but also the form and complexity of the shared dynamics of two systems. By reconstructing the possible states of the two systems and assessing the points in time in which they visit similar states, CRQA quantifies how often the two systems display similar patterns of change or movement, and how complex the structure of the entrainment between their trajectories is.

Several parameters have been suggested to articulate the structure of the coordination between two systems. Cross Recurrence Rate (RR) represents the "raw" amount of similarities between the trajectories of the two systems (the degree to which they tend to visit similar states). The structure of the similarities can be assessed along the diagonal and the vertical dimension. Diagonal structures represent periods in one time series that follow similar paths in their time-evolution to those in another time series, when aligned or shifted in time. The more closely coupled the two systems are, in terms of sharing the same paths, the more recurrences will be organized in diagonal lines. The measure that captures the rate of recurrence points forming diagonal lines is called determinism (DET) of the interaction between the two time series. The average length of the diagonal lines (L) represents the time that both systems stay attuned. The longest diagonal line on a recurrence plot (LMAX) represents the longest uninterrupted period of time that both systems stay attuned, which serves as an indicator of stability of the coordination: for example, sensitivity to noise and external perturbations creates unstable sequences of coordination and therefore a shorter longest diagonal line. It is also possible to measure how complex the attunement 
between the systems is (entropy or ENTR): if the diagonal lines tend to all have the same length the attunement is very regular (low ENTR), otherwise the attunement is complex (high ENTR). Finally, by analyzing which delay maximizes recurrence (diagonal recurrence profile, or DiagProfile), it is possible to observe the direction of the coordination, that is, if there is an asymmetry with one interlocutor leading the other. Diagonal structures thus highlight shared trajectories and their properties.

As an example, we simulated a strong and consistent coupling between two oscillators (i.e. the black and red time-series) in the line plot in Fig. 1a. Immediately below, the coupling can be observed in the cross-recurrence plot in the form of very evident diagonal structures. In Fig. 1b we introduced strong white Gaussian noise, creating two time-series with less stable coupling. This is reflected in the much weaker diagonal structures (fewer recurrences organized in diagonal lines and much shorter lines) in the cross-recurrence plot.

Vertical structures in a cross-recurrence plot quantify the propensity of the trajectories to stay in the same region (i.e. repeat the same value). In particular, the percentage of recurrence points forming vertical lines (as opposed to being isolated dots) is informative of the laminarity (LAM) of the interaction, and the average length of the vertical lines (trapping time, or TT) represents the average time two trajectories stay in the same region. As an example, in Fig. 1c we display the effects of stabilizing the two time-series at two moments (the flat lines in the line plot). The permanence of the trajectories in the same region (repeating the same value) is reflected in the vertical structures in the cross-recurrence plot.

While these examples are all of continuous variables, which have initially been the main focus of CRQA analyses, recent studies have developed ways to also explore the recurrence and cross recurrence of nominal sequences. These include sequences of phonemes, words, and coded behavior, such as the presence or absence of a particular gesture, or a nod [24, 30].

CRQA thus might constitute an answer to some of the issues posed by the analysis of social interactions: It enables the analysis of the shared dynamics of two time series. It does not assume stationarity and it is highly sensitive to the temporal structure of the interactions. It can cope with a wide variety of data, thus quantifying interactions between people in a wide range of modalities. Its output is highly articulated, allowing a fine-grained understanding of the structure of the shared dynamics between two agents. In the following section we investigate these potentialities, by critically reviewing the aspects of human interactions to which the method has been applied, and the picture that the results of the analyses enable us to sketch.

\section{CRQA and Social Interactions: From Swinging Pendula to Conversation}

A first crucial question to be answered is: can CRQA be used to assess the coupling between two systems (i.e. interpersonal coordination) at all? In other words, can it successfully assess the same dynamics assessed by other dynamical systems 
(a)

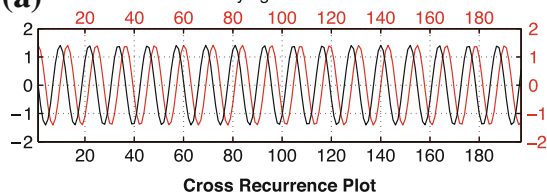

Dimension: 2, Delay: 4, Threshold: $0.1 \sigma$ (fixed distance maximum norm)

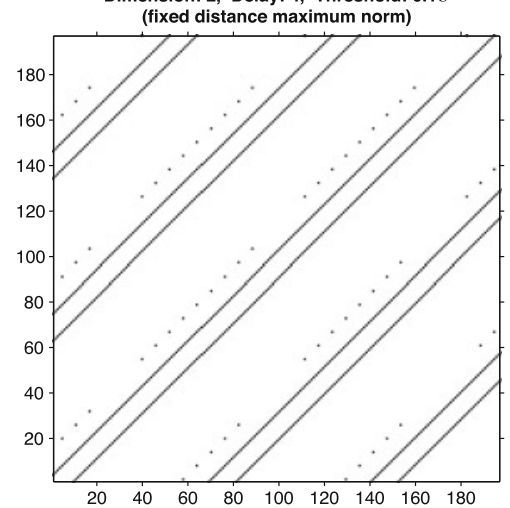

(b) Underlying Time Series

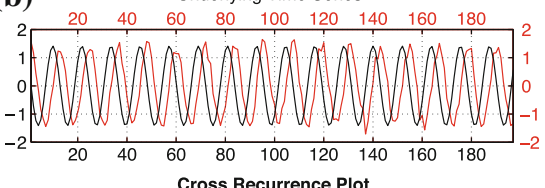

Dimension: 2, Delay: 4 , Threshold: $0.1 \sigma$

(fixed distance maximum norm)

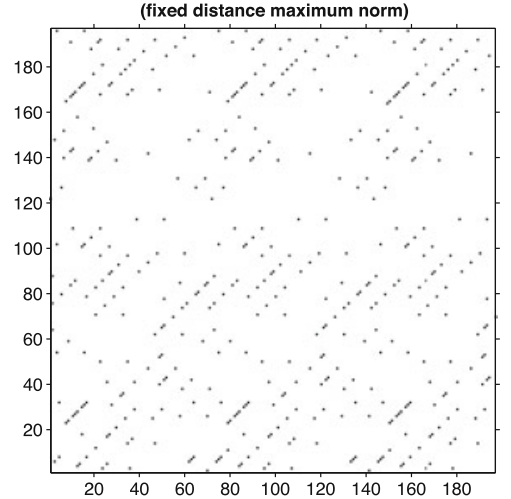

(c) Underlying Time Series

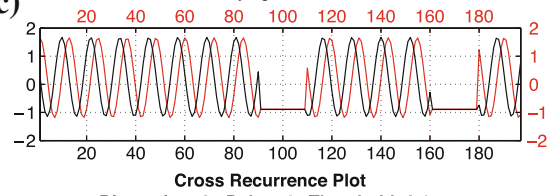

Dimension: 2, Delay: 4, Threshold: $0.1 \sigma$ (fixed distance maximum norm)

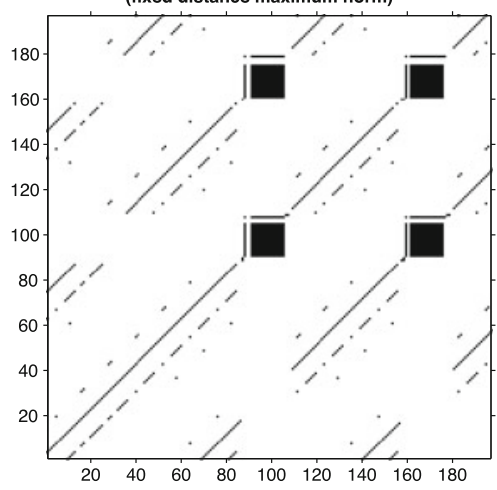

Fig. 1 Examples of diagonal and vertical recurrence structure. The plots were generated using the CRP toolbox for Matlab [29]

models, which are already widely used and very successful in capturing interpersonal phase and anti-phase synchronization [31-33]? In order to address these questions, Shockley et al. demonstrated the effectiveness of the method in assessing 
and quantifying coupling between two physical systems, above and beyond linear methods including cross-correlation and spectral analysis [34], by capturing shared dynamics using CRQA (quantified via both diagonal and vertical recurrence structures). It is thus no surprise that CRQA has been effectively employed to show basic synchronization phenomena between interacting agents. For example, people swinging pendula in a coordinated fashion show strong and stable coordination (high RR and LMAX, see [35]).

Given the complexity often present in any system's behaviors, it is, however, crucial that CRQA indexes are compared to an appropriate baseline, so to ensure that the degree of coordination they express is indeed due to coupling between the systems analyzed. A commonly employed baseline is the use of shuffled data, which maintains distributional properties but not the information contained in the temporal sequence of the data. In some cases, surrogate pairs are a more appropriate control condition, which consist of computing cross recurrence of time-series from mismatched pairs (e.g. matching the data from person A from pair 1 with the data from person B from pair 2). In surrogate pairs the overall individual structure is maintained, but the coupling dynamics are disrupted. The advantage of surrogate pairs is that they preserve the temporal organization of the overall event (e.g. the experimental task) as reflected in the individuals' time-series, but disrupt the actual dynamics between interacting agents. However, this control conditionis problematic in at least two cases: if the coordination analyzed involves turn-taking with alternate production of behavior of varying length(e.g. a conversation), the temporal structure of adjacent turns and the different lengths of the time-series would not be respected. A second problematic case is nominal time-series with sparse data, such as coded nodding, for which computer simulations have shown that shuffled data are a more conservative baseline [36]. Finally, a few studies have employed within pair contrasts: for example, in Ramenzoni et al. [37] the same pairs performed both interactive and noninteractive but otherwise similar tasks, with the non-interactive condition providing an ideal baseline. Analogously, Konvalinka et al. [38] compares the levels of heart rate coordination during a religious ritual with coordination before the ritual itself.

Once the sensitivity of CRQA to interpersonal coordination has been established against an appropriate baseline, the second question is: what does it add to a simpler phase analysis? CRQA is particularly useful for analyzing shared dynamics between signals that are not necessarily periodic in nature, or rather, whose periodic qualities are more complex, and hence relative phase analysis is not so straightforward. CRQA has thus been applied to many different aspects of complex interpersonal coordination, which would not be easily amenable to phase analysis, ranging from physiological to motor, linguistic and even conceptual ones.

A series of studies has shown how physiological signals, such as heart rate, also coordinate between individuals. Konvalinka et al. hypothesized physiological coordination to be involved in the community consolidating effects of highly arousing rituals. They investigated a fire walking ritual in Spain, showing that heart rhythms of firewalkers were more closely matched by heart rhythms of spectators who were their relatives or friends than those of non-related spectators, during the course of the ritual (higher RR, DET, LMAX, ENTR and LAM, see [38])—despite the fact that they did 
not have the same behavior. Fusaroli et al., in a less dramatic setting, investigated heart rate coordination in collective Lego construction tasks [39]. Groups of five participants built Lego models of abstract notions such as "trust" and "safety" alternatively as individuals ("Build your own individual model of trust") and collectively ("As a group build a model of trust that you all agree upon"). Interpersonal heart rate coordination (RR, L and ENTR) was shown to be significantly present in all groups both during individual and collective trials against shuffled baseline. However, a contrast with surrogate pairs showed no difference in individual trials - coordination being likely driven by task constraints as all participants in all groups were doing similar things - and higher levels of coordination during collective trials - coordination being likely driven by actual interactions.Not least, coordination during collective trials was shown to grow over time.

Focusing on motor coordination, Ramenzoni et al. assigned pairs of participants an interpersonal precision task: one participant holds a circle and the other has to keep a pointer inside the circle without touching its sides [11, 37, 40]. Hand and postural movements were strongly and stably coordinated across participants (RR and LMAX higher than in a non-interactive task), and increasing the difficulty of the task with smaller circles increased the coordinative structures. Analogously, stable coordinative structures were highlighted in groups of pedestrians walking in a crowded space (higher LMAX than control conditions, see [2]), and in duos and quartets of musicians and dancers [41, 42].

Interpersonal motor coordination appears very early in development: Reddy et al. investigated the specific structures of interpersonal coordination in infants anticipating being picked up [43]. Employing a pressure mat, the researchers showed that legs and arms of the infant are significantly coordinated (higher RR) with the mother already at 2 months of age, while full-bodied coordination appears at a later stage.

Notably, all of these studies have used high recurrence as the marker of coordination. However, Wallot et al. [44] present less straightforward findings. Pairs of participants built Lego cars together, while their hand-movements and heart rates were monitored. While significant behavioral and physiological coordinative structures were found (DET), they were negatively correlated with the effectiveness of the interaction measured in terms of functionality and aesthetic appeal of the resulting cars. These results might be interpreted as an effect of task constraint: doing the same thing might be counter-productive to effective collective construction, while distributing different subtasks - a division of labor strategy implying different actions for each participant - would be a better strategy.

Motor coordination has also been observed during conversations, where language acts as a highly effective social coordination device [16, 45, 46]. For instance, pairs of participants engaged in a joint problem-solving task show high coordination (RR and LMAX) in their postural sway, even when they are not looking at each other $[47,48]$. In a follow up experiment, Shockley et al. showed that postural sway coordination is mediated by two factors: interlocutors employing increasingly similar speech patterns, and actual interaction as opposed to simply repeating the same words in unison [49]. However, Richardson et al. report significant unintentional coordination (RR and LMAX) of hand-held pendula when pairs of participants solve 
a joint task while able to see each other, but where verbal interactions do not seem to have an effect [50]. This might be due to postural sway being a more natural part of linguistic interactions than swinging hand-held pendula.

While postural sway and hand-held pendula coordination might seem a simple byproduct of verbal coordination, or at most a facilitation of social rapport [51], other forms of coordinated behavior might have a more explicit functional role: for example, shared attention often relies on head movement and gaze coordination. It has been observed in several conversational contexts that interlocutors tend to coordinate gaze direction (high RR and low DiagProfile, [52]) and head movements (high RR, DET, L, LMAX, ENTR, LAM, TT, [53]), the latter which is mediated by dominance (higher scores for pairs with a dominant interlocutor) and gender (higher scores for women).

Gaze direction has been extensively explored in conversational scenarios by Richardson, Dale et al. The researchers recorded the speech and eye movements of one set of participants as they described pictures of six cast members of a TV sitcom. A second set of participants listened to these descriptions while looking at the same pictures. Gaze was highly coordinated between participants (RR), especially at a $2 \mathrm{~s}$ lag; in addition, the level of coordination correlated with the comprehension of the description [54]. When pairs of participants were asked to actively discuss pictures, the delay disappeared: they looked at the same elements at the same time [55]. In a third experiment, the researchers manipulated how much shared knowledge the participants had on the pictures to be discussed. Higher amount of shared knowledge generated higher coordination in eye movement [56]. In a final study, pairs of participants were presented with the same set of abstract shapes portrayed in different orders and they had to alternate in directing each other so that the orders would match. As the participants developed a common language to refer to these shapes, their eye-movements became increasingly coordinated at lag 0 , suggesting that they sampled the world in increasingly similar and effective ways [57]. Analogously, it has been shown that eye-movement recurrence is statistically higher when anaphoric and referential expressions are used [55, 58].In other words, specific linguistic items can be used as devices to further increase coordination. These results are supported by Jermann and Nussli's study, which reports increased gaze coordination (RR) in programmers jointly analyzing code, when they are allowed to talk and/or select portions of the text for each other [59]. In other words, by developing and implementing a shared language, participants develop effective interpersonal attention management systems [60]. These effects are not just limited to gaze or head movement coordination, but also includebehavioral matching of facial expressions, nodding, touching face, chin-resting, and manual gestures. Evidence for this comes from a systematic study investigating all of these behavioral measures during a task oriented conversation, where synchronous behavior matching was found across all the parameters (RR and DiagProfile) [36].

Up to now we have discussed how language facilitates motor coordination. However, the conversation itself also becomes coordinated between interlocutors as they manage turn-taking, adapt to each other's tone and language, construct shared routines, etc. The structure of turn-taking has been analyzed in many different ways: 
nominal sequences of $1 \mathrm{~s}$ (interlocutor speaking) and 0s (interlocutor being silent) [52, 53, 61, 62], employing utterance length [63, 64], or simply the sequence of interlocutors [65]. In all cases CRQA showed significant amounts of coordination (RR, cf. [52, 64]), tendency to keep attuned (DET [65]), synchronization (DiagProfile, [52, 62]) and in general diagonal and vertical recurrence structures [53, 63]. The amount of coordination has been reported to positively correlate with the experiential quality of the interaction [52, 64] and with the familiarity between interlocutors [65]. In other words, when we converse we (unsurprisingly) coordinate our utterances, this coordination is easier when we have familiar interlocutors, and the more coordinated we are the better the experience of the interaction.

From the developmental perspective, just as Reddy et al. [43] showed the coordinative adjustments in infants' movements, studies have demonstrated prosodic (fundamental frequency) coordination already from 3 months [66] and turn-taking coordination in children from at least 1 year of age [63]. However, turn-taking coordination seems to change in nature, being more rigid and repetitive (higher vertical structures) in younger children, and more flexible and extended (more prominent diagonal structure) in older ones [63]. Cognitive impairment (adults, [52]) and developmental disorders (children with autism, [62]) display a statistically lower amount of coordination and lack of immediate responsiveness (lower RR and higher DiagProfile). However, an exploratory study on adolescents with Moebius syndrome (involving congenital facial paralysis) shows high levels of conversational coordination in pitch and speech rate (RR, DET, L, LMAX), which decrease after an intervention aimed at improving social skills [67].

A few studies have also attempted to tackle the coordination of linguistic contents. Orsucci et al. [30, 68-70] tested CRQA of more symbolic aspects of language: they analyzedtranscripts of conversations by focusing on the recurrence of sequences of 3 characters roughly corresponding to morphemes. ${ }^{2}$ A natural conversation showed stronger coordinative patterns and attunement (RR and DET) than a clinical psychotherapy session, during which the patient's production tended to drift away from the therapist's. Dale and Spivey have investigated the coordination of syntactical patterns of words in children and caregivers engaged in naturalistic conversations (from the CHILDES corpus), reporting significant syntactic coordination (RR). While the coordination decreased as they grew up, it changed direction: initially the children followed adults, to increasingly assume leadership at later stages [71]. Louwerse et al. investigated the coordination of dialog acts (questions, answers, instructions, etc.), discourse connectives ("Alright", "ok", "hmm") and landmark descriptions in conversations where pairs of participants had to give each other instructions on how to draw a path on a map [36, 72]. Interestingly, they observed significant amounts of coordinative structures (RR and DiagProfile), which would decrease over time as the participants developed more minimal and effective ways to coordinate. However, when the difficulty of the task was increased, coordinative structures also increased.

\footnotetext{
2 The characters (including spaces) are converted to numbers, the embedding dimensions are set to 3 , and the threshold to 0 , to respect the categorical nature of the data.
} 
Fusaroli et al. analyzed several aspects of linguistic coordination at once in a series of task-oriented conversations [3, 73]. They analyzed coordination in turn-taking(as sequences of 1 and $0 \mathrm{~s}$ ), prosody (as fundamental frequency) and morphemes (as sequences of 3 characters) - and assessed it in relation to the efficacy of the conversations in enabling accurate solution of the task (a joint decision making). Pairs of participants showed consistent coordinative structures (L and ENTR)in all linguistic aspects analyzed, when compared to shuffled controls. However, the researchers argued that while CRQA quantifies the shared dynamics between participants, it might not capture other coordinative structures, for instance, complementary dynamics in which an interlocutor would share information only to beanswered by the other making a decision. Little alignment would be found there due to the different prosodic and lexical patterns involved in the two distinct conversational moves, but not for lack of tight coordination. These dynamics were better captured by employing RQA on the conversation as a whole without discriminating between interlocutors, and therefore highlighting how patterned and routinized the conversation became trial after trial. In a comparative analysis, while CRQA of prosody and morphemes positively correlated with performance, RQA of all aspects (turn-taking, prosody and morphemes) consistently provided better predictors of performance, with each aspect contributing non-overlapping information [61]. In other words, the best way to capture effective coordination was to look at how routinized the interactions became, making the decision making process as standardized, quick and efficient as possible. Those routines manifested at a lexical, prosodic and pause level. CRQA was not fully able to capture these complementary dynamics, but RQA was.

Angus et al. took an even more radical approach [74-77]. Being interested in the conceptual structure of conversations they organized the words used in the corpus in conceptual clusters, according to their co-occurrences within the same speechturns, or to pre-defined conceptual domains. They then analyzed the coordination of these conceptual clusters, defining new recurrence indexes taking into account the timescale (adjacent, mid-range, global), direction (backward and forward) and type (auto vs. cross recurrence). While still exploratory, these indexes of conceptual recurrence nicely characterized case studies including phone conversations, diagnostic interviews in clinical settings, and aircraft transcripts.

\section{Challenges and Recommendations}

We have shown that CRQA is suitable for analyzing many different aspects of coordination, from low-level physiological and motor synchronization, to complex joint actions and even symbolic and conceptual aspects of conversations. While several papers report case studies (11 out of 35), or limited numbers of participants, an increasing amount is scaling up the use of CRQA to statistically relevant samples (18 out of 35). The most basic indexes of recurrence-RR, DiagProfile and LMAXhave been shown sensitive to a wide range of conditions - such as gender, age, dominance, familiarity, modality of interaction and difficulty of the interaction-and to 
be reflected in the experience of the interaction. Other indexes-such as DET, L, ENTR, TT and LAM - are more sparsely employed, making it difficult to produce a clear picture of their relevance and meaning.

The most common result is that social interactions display higher amount and structure of recurrence than controls; however, a few isolated findings present more nuanced or even opposite findings, which seem worthy of further development and testing (cf. Challenge 2). We argue that the studies have demonstrated great promise for using CRQA to study social interactions, but that this analysis in relation to interaction is still in its birth, and we have only begun to ask many of the relevant questions. In particular we feel that the field faces 7 crucial challenges:

Challenge 1: to use CRQA when it is most appropriate and make its advantages explicit. Many researchers in psychology have shied away from applying CRQA to their data, even when working with continuous time-series that exhibit a non-linear, non-stationary structure. CRQA is a powerful but complex tool for the analysis of interaction data with an initial steep learning curve, and it has an articulated output that is often tricky to interpret. Thus, it might seem overly and unnecessarily complicated to scientists trained in more traditional methods. While it has provencapable of capturing basic periodic coordination (an important result to establish the validity of the method), for time-series that are predominantly linear in nature it might be more appropriate to use simple correlation-based analysis. Similarly, for quantifying nonlinear coupling between certain periodic signals, phase analysis might be sufficient. Not by chance, recent research on basic synchronization skills in clinical populations has been conducted with analysis of phase and coupled oscillators models by authors well versed in CRQA [31,33, 78]. While perfectly able to analyze these phenomena, CRQA gives its best on more complex and noisy data, which make it easier to justify its use. By more explicitly making CRQA one of the tools in the scientist's toolbox, arguing for its well-aimed use and showcasing its advantages over linear models for complex noisy data, it will be easier to get it accepted in mainstream research.

Challenge 2: to complement exploratory studies with theory-driven ones. This second challenge is shared with much of the investigations in social interactions. It is an informative and necessary step to explore the behavior of CRQA indexes in different coordinative contexts and on different aspects of coordination. However, such exploration should lead to theory-driven studies.

A first step in this direction is a more systematic exploration and report of indexes of cross recurrence across different types of interactions and modalities, which would be very useful in pinning down general patterns indicative of different coordination types. This could lead to the possibility of more fine-grained analyses in new contexts, where multiple CRQA indexes couldbe used to better assess the type of coordinative behavior-whether it is synchronized, stationary, and whether there are distinctive changes in coordinative structures throughout the interaction itself (i.e. mutual adaptation changing into leader-follower dynamics). In parallel, researchers should more consistently report their results: not only report all CRQA indexes to facilitate a more fine-grained grasping of the coordinative dynamics involved, but also effect sizes and statistical power (currently missing from the large majority of the studies reviewed). 
This would enable an easier planning of follow-up studies and facilitate cumulative science [79-82].

However, a more substantial step is the construction of conceptualmodels of coordination, which could generate hypotheses as to which recurrence indexes would be impacted by a manipulation, in which direction and, possibly, which range of effect sizes would be interesting. While RQA/CRQA has been really useful in analyzing non-linear dynamics across various modalities of human interaction, its interpretation and impact is sometimes unclear given the exploratory nature of most of these studies. For example, finding stronger coordination (i.e. higher RR, DET, LMAX) between certain motor activities of two people engaged in an interaction versus not interacting is interesting, but not particularly surprising. How can we use this information to make predictions about future behavior, about the outcome of the interaction, or for example, about the strength of rapport between two people? Some preliminary ideas are suggested by the studies reviewed, others by ongoing conceptual reflections in the field of interactions studies. For instance, a few studies seem to suggest that vertical structure and consistent delay in DiagProfile are related to simpler and more hierarchical interactions, while diagonal structures and low or alternating delay could be related to fluid, bidirectional, and flexible interactions. A second line of results questions the straightforward relation between amount and structure of recurrence and successful coordination, which is often assumed. In parallel, more conceptual studies have been developing the idea of alignment in social interactions as only one of the mechanisms at play, especially useful to initially establish the coordination and later to signal and repair problems, or even reinforce coordination if the difficulty of the task increases [83]. However, many forms of coordination include complementary dynamics, roles, and routines, which would require more nuanced analyses and could involve a decrease in recurrence diagonal structures. Thus, CRQA indexes might not increase with more fluent coordination and might not necessarily correlate with performance. This model could be used to hypothesize high presence of diagonal structures at the beginning of an interaction, which would decrease over time.

These are only initial suggestions: the study of social interactions in generalneeds more explicit models of coordination, and empirical investigations developed to assess and compare them $[6,10]$.

Challenge 3: to take into account multiple aspects of coordination at once and their interdependence. Studies like the ones performed by Louwerse [36], Fusaroli et al. $[39,61]$ are promising in these respects in that they systematically investigate several aspects of coordination at once and offer the possibility to map their interconnections. Dale and Louwerse are exploring how the many coordinated aspects of interpersonal behavior highlighted in their previous study relate to each other [72]. Analogously, Fusaroli et al. are exploring the connections between speech, actions, and heart rate coordination to understand what are the means through which physiological coordination is achieved [39]. It might be expected that not all aspects of coordination behave equally: for instance, achieving high levels of shared dynamics in motor behavior might create the enough common ground to enable interlocutors to effectivelydiversify their linguistic behavior [36], or vice versa highly shared 
linguistic dynamics might make complementary actions possible [44]. In any case, multivariate analyses of the multiple aspects seem necessary to account for many aspects of coordination at once $[84,85]$.

This might seem at odd with the previous challenge (more theory-driven studies to complement the explorative ones). We argue that it is possible to design a theorydriven experiment, with precise hypotheses, and complement it with exploratory analyses of a wider range of coordinative aspects and CRQA indexes, which will help generate new hypotheses for further studies.

Challenge 4: to account for multiple forms of coordination: complementarity and routines. Interestingly, the studies mentioned in the previous challenge also raise additional important issues for the study of coordination and the use of CRQA: In Louwerse et al., while the recurrence rate of many behaviors tends to grow over time, it decreases for others, in particular for language. In Fusaroli et al. CRQA parameters are a much worse index of effective coordination than Recurrence Quantification Analysis of the overall conversation [61]. Several other studies have shown that complementarity (rather than symmetry in action) is crucial for facilitation of coordination, as well as action understanding [10, 11, 21, 86, 87]. For example, when trying to move a table from one room to another, two people might produce complementary movements in order to more effectively achieve this goal—one person faces away from the table, grasping it with their hands behind them, while the other grasps it with their hands in front of them, facing the table [88]. Similarly, complementarity was encountered in a study where participants moved a marble in one direction by either one participant holding her edge of the tablet while the other is lifting it or one participant lowering her edge of the tablet while the other one is lifting it [89]. A cross-recurrence plot of the hand-accelerationsof participants, which was the dependent measure in this study, would either show strong cross-recurrence (when one participant would accelerate by lowering the tablet and the other one would accelerate by lifting the tablet) or weak cross-recurrence (when one would accelerate by lifting the tablet, but the other one would hold her hands still). In this case, an increase in cross recurrence structure does not equate to better coordination, but a more nuanced and task-specific understanding of coordinative structures has to be produced.

Fusaroli et al. [61] argued that in conversations and other kinds of coordination characterized by turn-taking complementarity might be captured by running RQA on pooled data from both participants: e.g. the whole conversation, without discriminating between interlocutors. Unfortunately, this does not seem a viable solution for assessing complementarity in tightly coupled motor interactions, where all agents continuously produce behavior. Whether CRQA can be developed to address complementarity in interaction remains an open question.

Challenge 5: to better understand how individual behaviors affect interpersonal coordination (and viceversa). Many studies have investigated the distinctive behaviors of people with mental and developmental disorders. For instance, RQA has been effectively used to characterize the distinctive speech patterns of people with autism, schizophrenia, depression and right hemisphere damage [90-93]. However, there is no model to understand how such individual patterns impact conversations and are 
therefore related to the social impairment these patients experience. More investigations and methodological development are needed to build more articulated models of coordination and advance our understanding in these fields.

Challenge 6: to account for multiple time scales at play in the interaction. Social interactions include processes and phenomena happening at many time scales [94, 95]. Continuous reciprocal adaptation might be a necessity when initiating an interaction and learning to coordinate with each other. However, interacting agents might gradually stabilize conventions such as local routines and even employ socially established scripts. How do we take these numerous time-scales into account? Angus et al. have developed interesting measures of conceptual recurrence reflecting short, mid and long range coordination [74]. More general forms of multi-scale recurrence quantification analysis have only recently started to be developed, but they might be crucial in solving these issues [96-100].

Challenge 7: to analyze interactions with more than two participants. Social interaction often involves more than two participants. Most current studies split the groups in sets of dyads [38, 39] and one uses aggregative measures [2]. It is an open challenge to preserve the group dynamics. Joint recurrence, network theory and probabilistic graphical models could provide ways to do so.

While many of these challenges require conceptual and methodological development, we advance some recommendations (often applying to the study of social interactions in general, irrespective of the methods employed), which would help developing the field:

- When possible attempt theory-driven predictions to identify relevant aspects of behavior, relevant recurrence indexes, and direction and size of the effect hypothesized. These predictions should take into consideration the form of coordination required by the task employed: for instance a task encouragingdifferential roles between the participantsmight yield less cross recurrence and diagonal structures when effectively coordinated than ineffectively. In this case, CRQA might not be the best method to use, as it would be difficult to tell whether less recurrence is because of weaker coupling, or complementary movements. On the contrary, a task based on similar roles would imply effective coordination with high levels of diagonal structures.Also, it would be useful to specify at which time-scale one would expect shared dynamics, as initial short-term alignment might be replaced by complementary roles and only be visible on longer time-scales as the participants switch roles.

- Systematically use control conditions and appropriate baselines. Since CRQA involves defining optimal parameters for each dataset (e.g. via normalizing and thresholding procedures), it is not always clear what constitutes statistically significant synchronization/coordination. Hence, appropriate control conditions should be designed, or failing that shuffled surrogates and false-pair-surrogates should be employed.

- Support the development of a finer grasp of the coordinative structures observed by reporting analyses on all recurrence indexes and not only the most common or the significant ones. When reporting results add a paragraph integrating the pattern of 
effects across the measures, which might help specifying which particular aspects of an interaction contributed to an outcome. For instance, an increase in $\mathrm{L}$ and LMAX could suggest that the duration of only one of many differenttime intervals during the interaction is crucial for the outcome, while an increase in L without a substantial increase in LMAX could be suggestive of a more systematic back-andforth in an interaction, where a clear alternation between behaviors is crucial.If necessary, less theory-drivenanalyses and interpretationscouldbe reported in the appendixes/supplementary materials.

- Support cumulative research and reproducibility, by calculating and reporting effect size and statistical power. Also, when possible, apply more advanced statistical methods, such as resampling methods to RQA/CRQA parameters (jackknifing, bootstrapping) to better estimate statistical precision [101].

\section{Conclusions}

In general, CRQA shows great promise for better understanding of the multiple timescales and parameters underlying social interactions. Important groundwork has been performed on a wide range of interpersonal phenomena: from physiological synchronization to complex joint actions and conversations. In this paper we have delineated seven crucial challenges and suggested a few recommendations to further develop the field. We believe that cumulative and theory-driven approaches, the analysis of complementarity,and more-than-two-agent interactions are some of the main challenges CRQA is still facing in its application to the study of social interaction.

Acknowledgments This research is supported by the Danish Council for Independent ResearchHumanities \& Technology and Production Sciences, the Interacting Minds Center (Aarhus University), the ERC Marie Curie Training Network Towards an Embodied Science of Intersubjectivity (TESIS) and the EUROCORES project: Digging the Roots For Understanding (DRUST).

\section{References}

1. Kimmel, M.: Intersubjectivity at close quarters: how dancers of Tango Argentino use imagery for interaction and improvisation. J. Cogn. Semiot. 4, 76-124 (2012)

2. Kiefer, A.W., et al.: Quantifying the coherence of pedestrian groups. in Cog Sci 2013, Berlin (2013)

3. Fusaroli, R., et al.: Coming to terms: an experimental quantification of the coordinative benefits of linguistic interaction. Psychol. Sci. 23(8), 931-939 (2012)

4. Hutchins, E.: How a cockpit remembers its speeds. Cogn. Sci. 19(3), 265-288 (1995)

5. Miller, J.H., Page, S.E.: Complex Adaptive Systems: An Introduction to Computational Models of Social Life. Princeton Studies in Complexity. Princeton University Press, Princeton (2007)

6. Dale, R., et al.: The self-organization of human interaction. Psychol. Learn. Motiv. 59, 43-95 (2013) 
7. Hasson, U., et al.: Brain-to-brain coupling: a mechanism for creating and sharing a social world. Trends Cogn. Sci. 16(2), 114-121 (2012)

8. Steptoe, A., et al.: Social isolation, loneliness, and all-cause mortality in older men and women. PNAS 110(15), 5797-5801 (2013)

9. Van Orden, G.C., Holden, J.G., Turvey, M.T.: Self-organization of cognitive performance. J. Exp. Psychol. Gen. 132(3), 331 (2003)

10. Fusaroli, R., Raczaszek-Leonardi, J., Tylén, K.: Dialog as interpersonal synergy. N. Ideas Psychol. 32, 147-157 (2014)

11. Riley, M.A., et al.: Interpersonal synergies. Frontiers Psychol. 2, 38 (2011)

12. Konvalinka, I., Roepstorff, A.: The two-brain approach: how can mutually interacting brains teach us something about social interaction? Frontiers Human Neurosci. 6, 1 (2012)

13. Bakeman, R., Gottman, J.: Observing Interaction: An Introduction to Sequential Analysis, 2nd edn. Cambridge University Press, Cambridge (1997)

14. Chartrand, T.L., Bargh, J.A.: The chameleon effect: the perception-behavior link and social interaction. J. Pers. Soc. Psychol. 76(6), 893-910 (1999)

15. Pickering, M.J., Garrod, S.: Toward a mechanistic psychology of dialogue. Behav. Brain Sci. 27(02), 169-190 (2004)

16. Fusaroli, R., Tylén, K.: Carving Language for Social Coordination: a dynamic approach Interaction studies 13(1), 103-123 (2012)

17. Sacheli, L.M., et al.: Kinematics fingerprints of leader and follower role-taking during cooperative joint actions. Exp. Brain Res. 226, 473-486 (2013)

18. Skewes, J., et al.: Implicit negotiation of leader-follower dynamics in an asymmetric joint aiming task. (Under revision)

19. Noy, L., Dekel, E., Alon, U.: The mirror game as a paradigm for studying the dynamics of two people improvising motion together. PNAS 108(52), 20947-20952 (2011)

20. Sebanz, N., Bekkering, H., Knoblich, G.: Joint action: bodies and minds moving together. Trends Cogn. Sci. 10(2), 70-76 (2006)

21. Masumoto, J., Inui, N.: Two heads are better than one: both complementary and synchronous strategies facilitate joint action. J. Neurophysiol. 109(5), 1307-1314 (2013)

22. Mills, G.: Dialogue in joint activity: complementarity, convergence and conventionalization. N. Ideas Psychol. 32, 158-173 (2014)

23. Vilar, L., et al.: Coordination tendencies are shaped by attacker and defender interactions with the goal and the ball in futsal. Hum. Mov. Sci. 33, 14-24 (2014)

24. Dale, R., Warlaumont, A.S., Richardson, D.C.: Nominal cross recurrence as a generalized lag sequential analysis for behavioral streams. Int. J. Bifurcat. Chaos 21, 1153-1161 (2011)

25. Coco, M.I., Dale, R.: Cross-recurrence quantification analysis of categorical and continuous time series: an R package. arXiv preprint arXiv:1310.0201, (2013)

26. Zbilut, J.P., Giuliani, A., Webber, C.L.: Detecting deterministic signals in exceptionally noisy environments using cross-recurrence quantification. Phys. Lett. A 246(1), 122-128 (1998)

27. Marwan, N., et al.: Recurrence plots for the analysis of complex systems. Phys. Rep. 438(5-6), 237-329 (2007)

28. Takens, F., Rand, D.A., Young, L.S.: Detecting Strange Attractors in Turbulence, in Dynamical Systems and Turbulence, pp. 366-381. Springer, Berlin (1981)

29. Marwan, N.: Cross recurrence plot toolbox. Available at http://tocsy.pik-potsdam.de/crp.php (2012)

30. Orsucci, F., Giuliani, A., Webber, C.: Combinatorics and synchronization in natural semiotics. Physica A: Stat. Mech. Appl. 361(2), 665-676 (2006)

31. Isenhower, R.W., et al.: Rhythmic bimanual coordination is impaired in young children with autism spectrum disorder. Res. Autism Spectr. Disord. 6(1), 25-31 (2012)

32. Kelso, J.A.S.: Dynamic Patterns: The Self-organization of Brain and Behavior. MIT Press, Cambridge (1995). (4 p. of plates)

33. Richardson, M.J., et al.: Rocking together: dynamics of intentional and unintentional interpersonal coordination. Hum. Mov. Sci. 26(6), 867-891 (2007) 
34. Shockley, K., et al.: Cross recurrence quantification of coupled oscillators. Phys. Lett. A 305(1-2), 59-69 (2002)

35. Richardson, M.J., et al.: Comparing the attractor strength of intra- and interpersonal interlimb coordination using cross-recurrence analysis. Neurosci. Lett. 438(3), 340-345 (2008)

36. Louwerse, M.M., et al.: Behavior matching in multimodal communication is synchronized. Cogn. Sci. 36(8), 1404-1426 (2012)

37. Ramenzoni, V.C., et al.: Joint action in a cooperative precision task: nested processes of intrapersonal and interpersonal coordination. Exp. Brain Res. Experimentelle Hirnforschung. Experimentation Cerebrale 211(3-4), 447-457 (2011)

38. Konvalinka, I., et al.: Synchronized arousal between performers and related spectators in a fire-walking ritual. PNAS 108(20), 8514-8519 (2011)

39. Fusaroli, R., Bjørndahl, J., Tylén, K.: A Heart for Coordination: investigating speech, gesture and heart rate in a collective, creative construction task. submitted

40. Ramenzoni, V.C., et al.: Interpersonal and intrapersonal coordinative modes for joint and single task performance. Human Movement Science, 2012

41. Varni, G., et al.: Toward a real-time automated measure of empathy and dominance. In: IEEE International Conference on Computational Science and Engineering, CSE'09 (2009)

42. Varni, G., Volpe, G., Camurri, A.: A system for real-time multimodal analysis of nonverbal affective social interaction in user-centric media. IEEE Trans. Multimedia 12(6), 576-590 (2010)

43. Reddy, V., Markova, G., Wallot, S.: Anticipatory adjustments to being picked up in infancy. PLoS ONE 8(6), e65289 (2013)

44. Wallot, S., et al.: Division of labor as a cooperative strategy during complex joint action. submitted

45. Tylén, K., et al.: Language as a tool for interacting minds. Mind Lang. 25(1), 3-29 (2010)

46. Clark, H.H.: Using Language. Cambridge University Press, Cambridge (1996)

47. Shockley, K., Santana, M.V., Fowler, C.A.: Mutual interpersonal postural constraints are involved in cooperative conversation. J. Exp. Psychol. Hum. Percept. Perform 29(2), 326332 (2003)

48. Shockley, K., Richardson, D.C., Dale, R.: Conversation and coordinative structures. Topics Cogn. Sci. 1(2), 305-319 (2009)

49. Shockley, K., et al.: Articulatory constraints on interpersonal postural coordination. J. Exp. Psychol. Hum. Percept. Perform 33(1), 201-208 (2007)

50. Richardson, M.J., Marsh, K.L., Schmidt, R.C.: Effects of visual and verbal interaction on unintentional interpersonal coordination. J. Exp. Psychol. Hum. Percept. Perform. 31, 62-79 (2005)

51. Marsh, K.L., Richardson, M.J., Schmidt, R.C.: Social connection through joint action and interpersonal coordination. Topics Cogn. Sci. 1(2), 320-339 (2009)

52. Reuzel, E., et al.: Conversational synchronization in naturally occurring settings: a recurrencebased analysis of gaze directions and speech rhythms of staff and clients with intellectual disability. J. Nonverbal Behav. 37(4), 281-305 (2013)

53. Ashenfelter, K.T.: Simultaneous Analysis of Verbal and Nonverbal Data During Conversation| Symmetry and Turn-taking. University of Notre Dame, Indiana (2008)

54. Richardson, D.C., Dale, R.: Looking to understand: the coupling between speakers' and listeners' eye movements and its relationship to discourse comprehension. Cogn. Sci. 29(6), 1045-1060 (2005)

55. Richardson, D.C., Dale, R., Kirkham, N.Z.: The art of conversation is coordination: common ground and the coupling of eye movements during dialogue. Psychol. Sci. 18(5), 407-413 (2007)

56. Richardson, D.C., Dale, R., Tomlinson, J.M.: Conversation, gaze coordination, and beliefs about visual context. Cogn. Sci. 33(8), 1468-1482 (2009)

57. Dale, R., Kirkham, N.Z., Richardson, D.C.: The dynamics of reference and shared visual attention. Front. Psychol. 2, 355 (2011) 
58. Diderichsen, P.: Cross recurrence quantification analysis of indefinite anaphora in swedish dialog. An eye-tracking pilot experiment. In: Brandial'06: Proceedings of the 10th Workshop on the Semantics and Pragmatics of Dialogue (SemDial-10), Universitätsverlag Potsdam, Germany, 11-13 September 2006

59. Jermann, P., Nüssli, M.-A.: Effects of sharing text selections on gaze cross-recurrence and interaction quality in a pair programming task. In: Proceedings of the ACM 2012 Conference on Computer Supported Cooperative Work. ACM, (2012)

60. Fusaroli, R., Gangopadhyay, N., Tylén, K.: The dialogically extended mind: making a case for language as skilful intersubjective engagement. Cogn. Syst. Res. 29-30, 31-39 (2014)

61. Fusaroli, R., Tylén, K.: Individual behavior, interactive alignment or interpersonal synergy? A model-comparison study on linguistic dialog. underRevision

62. Warlaumont, A.S., et al.: Vocal interaction dynamics of children with and without autism. In: Proceedings of the 32nd Annual Conference of the Cognitive Science Society. TX: Cognitive Science Society, Austin, (2010)

63. Cox, R.F., van Dijk, M.: Microdevelopment in parent-child conversations: from global changes to flexibility. Ecol. Psychol. 25(3), 304-315 (2013)

64. Rączaszek-Leonardi, J., et al.: Linguistic interaction as coordinative structure: Relationship between supraindividual and subjective. submitted

65. Gorman, J.C., et al.: Measuring patterns in team interaction sequences using a discrete recurrence approach. Hum. Factors: J Hum. Factors Ergon. Soc. 54(4), 503-517 (2012)

66. Buder, E.H., et al.: Dynamic indicators of mother-infant prosodic and illocutionary coordination. In: Proceedings of the 5th International Conference on Speech Prosody, (2010)

67. Michael, J., et al.: Compensatory Strategies Enhance Rapport in Interactions Involving People with Möbius Syndrome. submitted

68. Orsucci, F., Giuliani, A., Zbilut, J.: Structure \& coupling of semiotic sets. Experimental Chaos 742, 83-93 (2004)

69. Orsucci, F., et al.: Prosody and synchronization in cognitive neuroscience. EPJ Nonlinear Biomed. Phys. 1(1), 1-11 (2013)

70. Orsucci, F., et al.: Orthographic structuring of human speech and texts: linguistic application of recurrence quantification analysis. Arxiv preprint cmp-lg/9712010 (1997)

71. Dale, R., Spivey, M.J.: Unraveling the dyad: using recurrence analysis to explore patterns of syntactic coordination between children and caregivers in conversation. Lang. Learn. 56(3), 391-430 (2006)

72. Dale, R., Louwerse. M.M.: Human interaction as a multimodal network structure. In: Conceptual Structures, Discourse, and Language (2013)

73. Bahrami, B., et al.: Optimally interacting minds. Science 329, 1081-1085 (2010)

74. Angus, D., Smith, A., Wiles, J.: Human communication as coupled time series: quantifying multi-participant recurrence. IEEE Trans. Audio Speech Lang. Process. 20, 1795-1807 (2012)

75. Angus, D., Smith, A., Wiles, J.: Conceptual recurrence plots: revealing patterns in human discourse. IEEE Trans. Visual Comput. Graphics 18(6), 988-997 (2012)

76. Angus, D., et al.: Visualising conversation structure across time: insights into effective doctorpatient consultations. PloS ONE 7(6), e38014 (2012)

77. Leonardi, G.: The study of language and conversation with recurrence analysis methods. Psychol. Lang. Commun. 16(2), 165-183 (2012)

78. Marsh, K.L., et al.: Autism and social disconnection in interpersonal rocking. Frontiers Integr. Neurosci. 7, 4 (2013)

79. Lakens, D.: Calculating and reporting effect sizes to facilitate cumulative science: a practical primer for t-tests and anovas. Frontiers Psychol. 4, 863 (2013)

80. Dienes, Z.: Understanding Psychology as a Science : An Introduction to Scientific and Statistical Inference, p. 170. Palgrave Macmillan, New York (2008)

81. Plonsky, L.: In: Porte, G. (ed.) Replication, Meta-analysis, and Generalizability. Replication Research in Applied Linguistics. Cambridge University Press, Cambridge (2012)

82. Cumming, G.: The new statistics why and how. Psychol. Sci. 25(1), 7-29 (2014) 
83. Dale, R., et al.: Beyond synchrony: complementarity and asynchrony in joint action. In: Cognitive Science (2013)

84. Thomasson, N., Webber, C., Zbilut, J.P.: Application of recurrence quantification analysis to EEG signals. Int. J. Comput. Appl. 9, 9-14 (2002)

85. Marwan, N., Kurths, J., Saparin, P.: Generalised recurrence plot analysis for spatial data. Phys. Lett. A 360(4), 545-551 (2007)

86. Kokal, I., Gazzola, V., Keysers, C.: Acting together in and beyond the mirror neuron system. Neuroimage 47(4), 2046-2056 (2009)

87. Newman-Norlund, S.E., et al.: Recipient design in tacit communication. Cognition 111, 46-54 (2009)

88. Sebanz, N., Bekkering, H., Knoblich, G.: Joint action: bodies and minds moving together. Trends Cogn. Sci. 10(2), 70-76 (2006)

89. Lang, M., et al.: Lost in the rhythm: the effects of rhythm on subsequent interpersonal coordination. underRevision

90. Fusaroli, R., et al.: Non-linear dynamics of speech and voice in schizophrenia. in Neurobiology of Language 2013, San Diego (2013)

91. Fusaroli, R., Bang, D., Weed, E.: Non-linear analyses of speech and prosody in asperger's syndrome. In: IMFAR 2013, San Sebastian (2013)

92. Fusaroli, R., et al.: Non-linear dynamics of voice in mental disorders. in Cog Sci 2013, Berlin (2013)

93. Weed, E., Fusaroli, R.: Prosodic production in right-hemisphere stroke patients: using temporal dynamics to characterize voice quality. in Neurobiology of Language (2013)

94. Raczaszek-Leonardi, J., Kelso, J.A.S.: Reconciling symbolic and dynamic aspects of language: toward a dynamic psycholinguistics. N. Ideas Psychol. 26(2), 193-207 (2008)

95. Fusaroli, R., et al.: Conversation, coupling and complexity: matching scaling laws predict performance in a joint decision task. in Cog Sci 2013 (2013)

96. Chen, Y., Yang, H.: Multiscale recurrence analysis of long-term nonlinear and nonstationary time series. Chaos Solitons Fractals 45(7), 978-987 (2012)

97. Xiang, R., et al.: Multiscale characterization of recurrence-based phase space networks constructed from time series. Chaos 22(1), 013107 (2012)

98. Lancia, L., Avelino, H. Voigt, D.: Measuring laryngealization in running speech: interaction with contrastive tones in Yalálag Zapotec. In: Interspeech 2013. in press

99. Lancia, L., Fuchs, S., Tiede, M.: Application of concepts from cross-recurrence analysis in speech production: an overview and a comparison to other nonlinear methods. J. Speech Lang. Hearing Res. 57, 743-757 (2013)

100. Lancia, L., Tiede, M.: A survey of methods for the analysis of the temporal evolution of speech articulator trajectories. In: Fuchs, A., et al. (eds.) Speech Planning and Dynamics, Peter Lang (2012)

101. Schinkel, S., et al.: Confidence bounds of recurrence-based complexity measures. Phys. Lett. A 373(26), 2245-2250 (2009) 